\title{
Prompt optical observations of GRB 080330 and GRB 080413A
}

\author{
Yuan, F*, Rykoff, E. S. ${ }^{\dagger}$, Schaefer, B. E.**, Rujopakarn, W.*, Güver, T. ${ }^{\S}$, \\ Aharonian, F. ${ }^{\top}$, Akerlof, C. W. ${ }^{*}$ Ashley, M. C. B.", Barthelmy, S. D. ${ }^{\dagger}$,

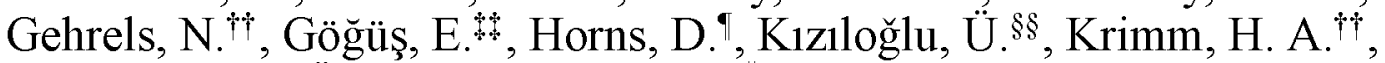 \\ McKay, T. A.*, Özel, M.`", Phillips, A.", Quimby, R. M.***, Rowell, G.",

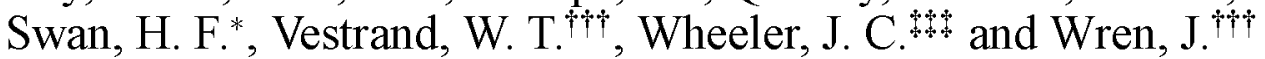 \\ *University of Michigan, 2477 Randall Laboratory, 450 Church St., Ann Arbor, MI, 48109 \\ $\dagger$ Physics Department, University of California at Santa Barbara, 2233B Broida Hall, Santa Barbara, CA 93106 \\ ${ }^{* *}$ Department of Physics and Astronomy, Louisiana State University, Baton Rouge, LA 70803 \\ * Steward Observatory, University of Arizona, Tucson, AZ 85721 \\ $\S$ University of Arizona, Tucson, AZ 85721 \\ ${ }^{\top}$ Max-Planck-Institut für Kernphysik, Saupfercheckweg 1, 69117 Heidelberg, Germany \\ "School of Physics, University of New South Wales, Sydney, NSW 2052, Australia \\ $\uparrow N A S A$ Goddard, Greenbelt, MD 20771 \\ *Sabancı University, Orhanl-Tuzla 34956 Istanbul, Turkey \\ $\$ \S$ Middle East Technical University, 06531 Ankara, Turkey \\ "IT Çanakkale Onsekiz Mart Üniversitesi, Terzioğlu 17020, Çanakkale, Turkey \\ ${ }^{* * *}$ Astronomy Department, Califomia Institute of Technology, 105-24, Pasadena, CA 91125 \\ ${ }^{\dagger \dagger}$ Los Alamos National Laboratory, NIS-2 MS D436, Los Alamos, NM 87545 \\ Hepartment of Astronomy, University of Texas, Austin, TX 78712
}

\begin{abstract}
We report on the results of rapid responses to GRB 080330 and GRB 080413A by the ROTSE-IIIb and ROTSEIIIc telecopes. In both cases, optical follow-up started during the prompt emission phase and recorded optical flares peaking at similar times as the last hard X-ray pulses detected by the Swift BAT. For both events, the XRT observations started at the end of the prompt BAT detection and displayed an evolution in time opposite to the optical lightcurve. These very early observations provide us an opportunity to probe the onset of afterglow for these two events.
\end{abstract}

Keywords: gamma-rays: bursts

PACS: $98.70 . \mathrm{Rz}$

\section{ROTSE-III: INTRODUCTION AND STATUS}

ROTSE-III is an array of four identical $0.45 \mathrm{~m}$ robotic telescopes installed at widely dispersed locations. Designed for rapid GRB response, these telescopes can slew quickly in $\sim 7$ seconds to any target position on the sky. At any time, at least one ROTSE-III telescope is in astronomical night. The systems are operated without filters to maximize the detection sensitivity. The typical limiting magnitude is $\sim 18.5$ for a 60 -seconds exposure. Details of the ROTSE-III telescopes are described in [1].

By end of July 2008, ROTSE-III has quickly responded to 95 Swift bursts ( $\sim 1 / 3$ of all triggers). For 38 events, imaging started before the end of the prompt emission, yielding 18 prompt optical detections.

\section{GRB 080330: RESPONSE AND LIGHT CURVE}

GRB 080330 was detected by the Swift BAT on 03:41:16.8 UT (trigger 308041, [2]). The burst had a $T_{90}$ duration of $61 \pm 9 \mathrm{~s}$ in the $15-350 \mathrm{keV}$ band, with a fluence of $(3.4 \pm 0.8) \times 10^{-7} \mathrm{erg} / \mathrm{cm}^{2}$ in the $15-150 \mathrm{keV}$ band [3]. The BAT lightcurve showed 3 overlapping peaks and a fourth peak seperated from the initial set by about $35 \mathrm{~s}$. Swift XRT and UVOT started observing the field 77 and 63 seconds after the trigger respectively and both detected the counterpart 

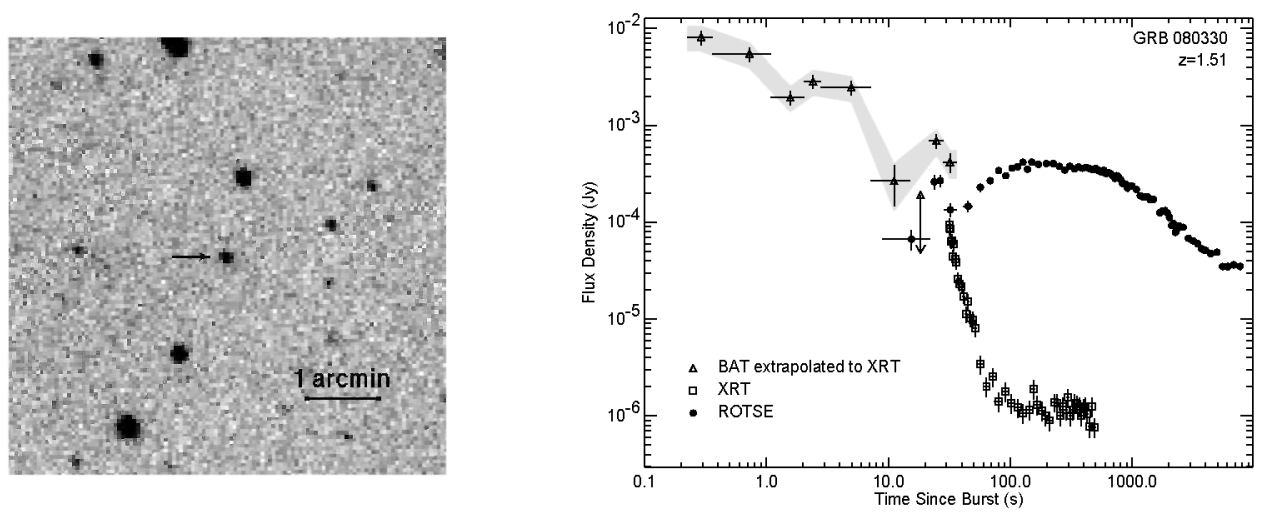

FIGURE 1. ROTSE image of the OT at $427 \mathrm{~s}$ after the burst and broad-band lightcurves of GRB 080330 in its rest frame.

[4]. [5] measured an absorption redshift of 1.51 for the event with the NOT Telescope. This was confirmed by [6] with the HET Telescope.

ROTSE-IIIb, at McDonald Observatory, Texas, responded automatically to the GCN notice. The first exposure started at 03:41:39.2 UT, 7.2 seconds after the notice, 22.4 seconds from the burst onset and before rising of the last pulse measured by BAT. The automated burst response included a set of ten $5 \mathrm{~s}$ exposures, ten $20 \mathrm{~s}$ exposures, and over 400 $60 \mathrm{~s}$ exposures. Near real-time analy sis of the ROTSE-III images detected a source at 11:17:04.5+30:37:24.0 (J2000.0) that was not visible on the Digitized Sky Survey red plates, as was reported via GCN 7538 [7]. This source was not detected in the first five single exposures, but it brightened to about $17.5 \mathrm{mag}$ in the 6th and 7 th images starting $57 \mathrm{~s}$ and $64 \mathrm{~s}$ after the burst. This first peak roughly coincide with the fourth peak in BAT. The optical counterpart declined rapidly and rose again to a second peak of about $17.3 \mathrm{mag}$ at about 300 seconds after the burst. The later observation is consistent with other optical detections of the afterglow reported in GCNs $[8,2,9,10]$.

Fig. 1. shows a ROTSE image of the optical counterpart and the ROTSE lightcurve plotted with the X-ray observations in the burst rest frame. ROTSE magnitudes are converted to flux density assuming equivalence to $R_{c}$ and have been approximately adjusted for Galactic extinction by $0.044 \mathrm{mag}$ [11]. Drifting of the spacecraft [12] complicated the XRT analysis and we therefore use the X-ray lightcurves from the Swift archive (http://www.swift.ac.uk/xrt_curves/). The BAT Data have been analyzed using the standard analysis software distributed within FTOOLS, version 6.4a and extrapolated to the XRT range using the X-ray spectral index reported in [4].

\section{GRB 080413A: RESPONSE AND LIGHTCURVE}

GRB 080413A was detected by the Swift BAT on 02:54:19.3 UT (trigger 309096, [13]). The burst had a $T_{90}$ duration of $46 \pm 1 \mathrm{~s}$ in the $15-350 \mathrm{keV}$ band with a fluence of $(3.5 \pm 0.1) \times 10^{-6} \mathrm{erg} / \mathrm{cm}^{2}$ in the $15-150 \mathrm{keV}$ band [14]). The BAT lightcurve showed two overlapping initial peaks and two more seperated peaks extending to $T_{0}+80 \mathrm{sec}$. Swift XRT and UVOT started observing 61 seconds after the trigger and both detected the counterpart [15]. [16] measured an absorption redshift of 2.433 for this event with UVES at the VLT. This was confirmed by [17] with the Gemini-South Telescope.

ROTSE-IIIc, at the H.E.S.S. site in Namibia, responded automatically to the GCN notice. The first exposure started at 02:54:39.7 UT, 6.7 seconds after the notice, 20.4 seconds from the burst onset and during decay of the third pulse measured by BAT. The automated burst response included a set of ten $5 \mathrm{~s}$ exposures, ten $20 \mathrm{~s}$ exposures, and $9060 \mathrm{~s}$ exposures before twilight. Near real-time analysis of the ROTSE-III images detected a 12.8 mag source at 19:09:11.8 $-27: 40: 41.6$ (J2000.0) that was not visible on the Digitized Sky Survey red plates, which was reported via GCN 7593 [18].

Fig. 2. shows a ROTSE image of the optical counterpart and the ROTSE lightcurve plotted with the X-ray observations in the burst rest frame. ROTSE magnitudes are converted to flux density assuming equivalence to $R_{c}$ and have been approximately adjusted for Galactic extinction by 0.415 mag [11]. The OT is 14 arcsec away from a 15.0 mag object, but is well resolved in ROTSE images. (Photometry may be affected when the OT faded below the brightness of the possibly contaminating source, so we will focus on the early part of the lightcurve). The BAT and XRT Data have 

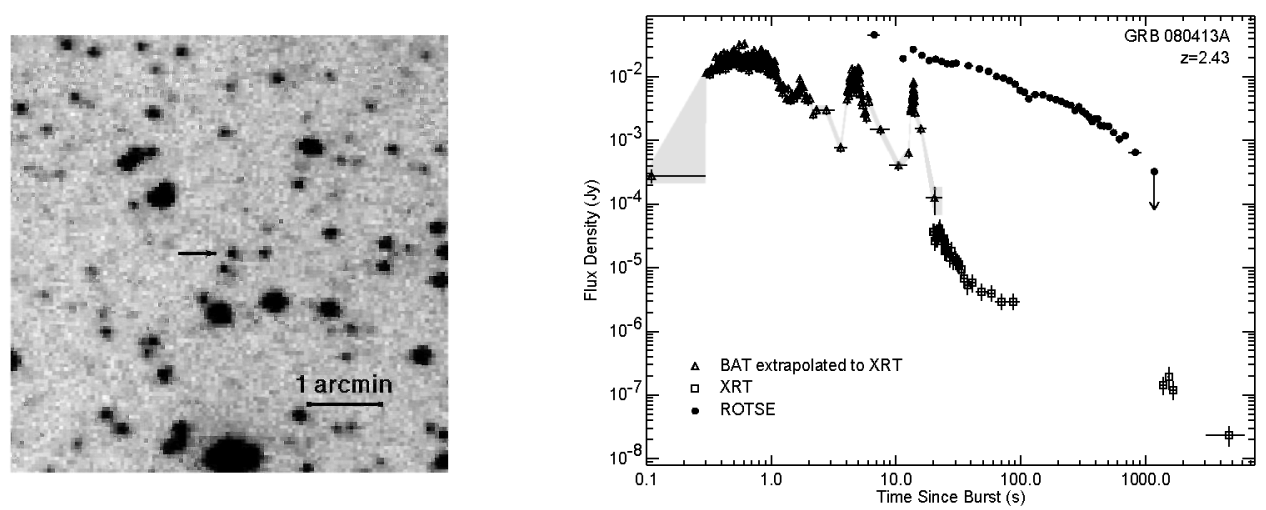

FIGURE 2. ROTSE image of the OT at $448 \mathrm{~s}$ after the burst and broad-band lighteurves of GRB $080413 \mathrm{~A}$ in its rest frame.

been analyzed using the standard analysis software distributed within FTOOLS, version $6.4 \mathrm{a}$ and the BAT lightcurve extrapolated to the XRT range using the X-ray spectral index consistent with that reported in [15].

\section{DISCUSSION}

For GRB 080330, the optical flare was followed by a relatively fast rise to a second optical emission peak. The lightcurve afterward show an initial very slow decay which then smoothly steepened. This plateau phase has a similar decay index ( $\sim-0.1)$ with the X-ray during the overlapping observation and may be explained as afterglow emission. The features of this lightcurve agree with that of a structured flow viewed off-axis, which is modeled in Figure 5 of [19].

The lightcurve of GRB 080413A is more complicated, but also shows a slow decay after the optical flare which smoothly steepened. The coincidence of optical and hard X-ray flares suggests that they share a common origin, e.g. internal activity. The later smooth optical lightcurve is likely to be of external origin and the rising of this afterglow phase may have been overwhelmed by the energetic 'prompt optical emission'. The initial decay inferred from the first two ROTSE data points doesn't have the temporal resolution to address correlation with the hard X-ray prompt emission. It certainly doesn't fit with back-extrapolation of the afterglow component but also seems to have an inconsistent optical to high energy ratio with the flare.

$\mathrm{X}$-ray lightcurve for this event again can be described by power-laws with a canonical steep to shallow break. Evidence of spectral evolution in X-ray [15] suggests the shallower part is from a different emission mechanism. Direct comparison with optical is limited by the temporal coverage.

Different peak times of the afterglow for the two bursts may be explained by difference in the initial Lorentz factor. According to equation 1 in [20], the rise time of the afterglow is more sensitive to the initial Lorentz factor than to the total energy release. The delayed peak time in the afterglow of GRB 080330 may point to a small initial Lorentz factor, which is consistent with this event being soft and classified as an X-ray Flash in terms of its fluence ratio in BAT high and low energy band [21,4]. Precise calculation for this event is not permitted due to the limited knowledge of the spectral shape from the BAT observation alone.

\section{CONCLUSION}

A diverse range of behaviors have been observed in optical lightcurves during the prompt gamma-ray emissions. Although the number of prompt optical detections are increasing thanks to the successful operation of Swift, wellsampled early observations are still technically difficult to achieve.

The very early optical observation may have components from both the prompt emission and the early afterglow, or, as in the standard fireball model, from both internal and external shocks. The internal emission mechanism is not well understood and the strength of this emission in optical band is so far not well constrained either by theory or by observation. Lightcurves of GRB 080330 and GRB 080413A show evidence that prompt optical emission can be 
comparable in magnitude to the afterglow peak and easily obscured if the afterglow rises quickly. It's clear that both components have to be considered when studying early optical observations.

For GRB 080330, the rising afterglow may be back-extrapolated and removed from the prompt emission phase. This gives us an opportunity to look at the real correlation between optical and hard x-ray. Apparently decay of the first optical peak then becomes much steeper than the decay of that pulse measured by BAT. However, poor sampling at this early time makes it difficult to draw a meaningful conclusion.

More energetic events like GRB 080413A provide a brighter optical counterpart and a chance of better sampling, but the afterglow also rises quickly and overlaps with the prompt emission. To effectively separate the contributions, better understanding of either region is required. Low initial Lorentz factor or geometric effects may delay the rise of the afterglow. Study of such late rising lightcurves, such as that observed for GRB 080330, may help to constrain the behavior of very early afterglow. In turn, prompt optical emission can be studied quantitatively for complex events like GRB 080413A. Study of more samples, both detections and limits are needed to address the details.

\section{ACKNOWLEDGMENTS}

This work is supported by NASA Swift Guest Investigator grants NNX-07AF02G and NNX-08AN25G. ROTSE-III has been supported by NASA grant NNG-04WC41G, the Australian Research Council, the University of New South Wales, the University of Texas, and the University of Michigan. Special thanks to the observatory staff at McDonald Observatory, especially David Doss. Special thanks to the H.E.S.S. staff, especially Toni Hanke.

\section{REFERENCES}

1. C. W. Akerlof, R. L. Kehoe, T. A. McKay, E. S. Rykoff, D. A. Smith, D. E. Casperson, K. E. McGowan, W. T. Vestrand, P. R. Wozniak, J. A. Wren, M. C. B. Ashley, M. A. Phillips, S. L. Marshall, H. W. Epps, and J. A. Schier, PASP 115, 132-140 (2003).

2. J. Mao, W. H. Baumgartner, D. N. Burrows, N. Gehrels, S. T. Holland, J. A. Kennea, K. M. McLean, D. M. Palmer, A. M. Parsons, M. Stamatikos, R. L. C. Starling, T. N. Ukwatta, and D. E. vanden Berk, GCN Circ. 7537 (2008).

3. C. Markwardt, S. D. Barthelmy, J. Cummings, E. Fenimore, N. Gehrels, H. Krimm, J. Mao, K. McLean, D. Palmer, A. Parsons, T. Sakamoto, G. Sato, M. Stamatikos, J. Tueller, and T. Ukwatta, GCN Circ. 7549 (2008)

4. J. Mao, C. Guidorzi, C. Markwardt, N. P. M. Kuin, S. D. Barthelmy, D. N. Burrows, P. Roming, and N. Gehrels, GCN Report $132.1(2008)$

5. D. Malesani, J. P. U. Fynbo, P. Jakobsson, P. M. Vreeswijk, and S.-M. Niemi, GCN Circ. 7544 (2008).

6. A. Cucchiara, and D. B. Fox, GCN Circ. 7547 (2008)

7. B. E. Schaefer, and T. Guver, GCN Circ. 7538 (2008)

8. A. Klotz, M. Boer, and J. L. Atteia, GCN Circ. 7536 (2008).

9. A. Gomboc, C. Guidorzi, A. Melandri, C. G. Mundell, A. Monfardini, R. J. Smith, I. A. Steele, D. Bersier, S. Kobayashi, M. Burgdorf, and D. Carter, GCN Circ. 7539 (2008).

10. A. Klotz, M. Boer, and J. L. Atteia, GCN Circ. 7543 (2008).

11. D. J. Schlegel, D. P. Finkbeiner, and M. Davis, ApJ 500, 525-553 (1998).

12. D. N. Burrows, GCN Circ. 7541 (2008).

13. A. P. Beardmore, D. N. Burrows, P. A. Evans, N. Gehrels, S. T. Holland, S. D. Hunsberger, J. A. Kennea, C. B. Markwardt, K. L. Page, D. M. Palmer, T. Sakamoto, M. Stamatikos, and E. Troja, GCN Circ. 7594 (2008).

14. J. Tueller, S. D. Barthelmy, W. Baumgartner, A. P. Beardmore, J. Cummings, E. Fenimore, N. Gehrels, H. Krimm, C. Markwardt, K. McLean, D. Palmer, T. Sakamoto, G. Sato, M. Stamatikos, and T. Ukwatta, GCN Circ. 7604 (2008).

15. F. E. Marshall, S. D. Barthelmy, D. N. Burrows, P. A. Evans, S. R. Oates, M. Stamatikos, and N. Gehrels, GCN Report 129.1 (2008).

16. C. C. Thoene, D. Malesani, P. M. Vreeswijk, J. P. U. Fynbo, P. Jakobsson, C. Ledoux, and A. Smette, GCN Circ. 7602 (2008).

17. A. Cucchiara, D. B. Fox, and S. B. Cenko, GCN Circ. 7616 (2008).

18. E. S. Rykoff, and W. Rujopakarn, GCN Circ. 7593 (2008).

19. A. Panaitescu, and W. T. Vestrand, MNRAS 387, 497-504 (2008).

20. E. Molinari, S. D. Vergani, D. Malesani, S. Covino, P. D’Avanzo, G. Chincarini, F. M. Zerbi, L. A. Antonelli, P. Conconi, V. Testa, G. Tosti, F. Vitali, F. D’Alessio, G. Malaspina, L. Nicastro, E. Palazzi, D. Guetta, S. Campana, P. Goldoni, N. Masetti, E. J. A. Meurs, A. Monfardini, L. Norci, E. Pian, S. Piranomonte, D. Rizzuto, M. Stefanon, L. Stella, G. Tagliaferri, P. A. Ward, G. Ihle, L. Gonzalez, A. Pizarro, P. Sinclaire, and J. Valenzuela, $A \& A$ 469, L13-L16 (2007).

21. T. Sakamoto, D. Hullinger, G. Sato, R. Yamazaki, L. Barbier, S. D. Barthelmy, J. R. Cummings, E. E. Fenimore, N. Gehrels, H. A. Krimm, D. Q. Lamb, C. B. Markwardt, J. P. Osborne, D. M. Palmer, A. M. Parsons, M. Stamatikos, and J. Tueller, ApJ 679, 570-586 (2008). 